\title{
OPEN STANDARDS AND OPEN SOURCE: ENABLING INTEROPERABILITY
}

\author{
Fernando Almeida ${ }^{1}$ José Oliveira $^{2}$ and José Cruz ${ }^{3}$ \\ ${ }^{1}$ Innovation and Development Center, ISPGaya, V.N.Gaia, Portugal \\ falmeida@ispgaya.pt \\ ${ }^{2}$ Faculty of Economics and INESC Porto, University of Porto, Porto, Portugal \\ jmodinescporto.pt \\ ${ }^{3}$ Faculty of Engineering, University of Porto, Porto, Portugal \\ jmcruzefe.up.pt
}

\begin{abstract}
Interoperability is a major requirement for industries and governments in a society that increasingly moves towards global collaboration and integration. Open standards built on the principles of openness, transparency and consensus lay the grounds for innovation, growth and fair competition. Open standards are not synonymous of open source. The former is a set of specifications, the latter is an implementation. However, they share their commitment to openness and defend the equal opportunities of everyone to participate. This paper looks to the open source as the best way to enable interoperability between different technologies and applications. The role of open standards in interoperability is analyzed and some of the policies introduced by the European Union for the use and dissemination inside Members States are examined. Additionally, the use of open source software combined with open standards is presented and its major social benefits and economic impacts are highlighted.
\end{abstract}

\section{KEYWORDS}

Open Standards, Open Source, Interoperability, Software Development

\section{INTRODUCTION}

Interoperability is an enabler for the growth of software (and also hardware) industries. Any software company is able to produce both large and small applications and utilities, competing in the open market if it know that its products co-exist and connect with other applications or pieces of software already developed and available, or even future similar products. That ability of co-existence and interconnection is what interoperability is all about and the World Wide Web is the main proof of the importance of the concept. Interoperability itself becomes a standard.

Open source development has been around several years and is renowned for its extraordinary code quality [1]. Some of the more popular open source projects include Linux, Apache and MySQL. Additionally to its non-traditional distribution model that relies on software downloads from a website, the development of software code is collaborative and not handled by a single company but by a community of high-skilled, fascinated and encouraged developers. The community may be comprised of companies and/or individuals. The licensing models typically used promote the sharing and reuse of software source code with relaxed intellectual property restrictions.

Open source development makes particular sense in the area of interoperability. That is because the best way to build things that work together is when group of people or companies also work collectively, at the code level, creating something they can all use. Proprietary approaches and 
International Journal of Software Engineering \& Applications (IJSEA), Vol.2, No.1, January 2011

solutions drive up the cost of development which translates into a higher cost to the consumer. Several industries, such as telecommunications and information technologies already leveraged and embraced open source technologies to improve quality and reduce costs [2]. Furthermore, customers dislike vendor lock-in in critical solutions components in their business applications.

As indicated in 2008 by Deshpande and Riedhle [3], Open source software today has a strong presence in industry and government. Walli, et al. [4] observed that worldwide organizations are saving millions of dollars on IT by using open source software. In 2004, large companies (with annual revenue of over $\$ 1$ billion) saved an average of $\$ 3.3$ million using open source software. Medium-sized companies (between $\$ 50$ million and $\$ 1$ billion in annual revenue) saved an average of $\$ 1.1$ million. Firms with revenues under $\$ 50$ million saved an average of $\$ 520$ thousands [5]. Commercially, the significance and growth of open source is measured in terms of revenue generated from it. IDC also states that packaged open source applications generated revenues of $\$ 1.8$ billion in 2006 [5]. The software division of the Software \& Information Industry Association estimates that total packaged software revenues were \$235 billion in 2006 [6]. Thus, open source revenue, while still small compared to the overall market (around $0.7 \%$ ) is not trivial any longer.

IDC has labelled open source "the most significant all-encompassing and long-term trend that the software industry has seen since the early 1980s". Worldwide revenue from open source solutions is expected to reach $\$ 5.8$ billion by 2011 , a notable growth rate of more than $26 \%$ [5]. Gartner also predicts that by 2012 , more than $90 \%$ of enterprises will use open source in direct or embedded forms and the majority of the software-as-a-service deployments will be open source. In the same study, Gartner advises that developers, whether in vendors or user organizations, should not consider taking on new interoperability challenges with new code unless open-source alternatives do not exist or have failed after a diligent attempt to make them work.

\section{THE ROLE OF OPEN STANDARDS IN INTEROPERABILITY}

\subsection{Definition of open standards}

The word "standard" has several different meanings. Within the context of this article, its meaning can be taken to refer to a level of quality or attainment, or an item or a specification against which all others may be measured. In technical usage, a standard is a framework of specifications that has been approved by a recognized organization, or is generally accepted and widely used throughout by the industry [7].

However, the concept of open standard is far less linear. In reality, there are many different opinions on what represents open standards.

Bruce Perens, a recognized open source specialist, argues that an open standard is more than just a specification, and that the principles underlying the standard and the practice of offering and operating it are what makes the standard open [8]. He defends that open standards should follow the principles of availability and maximizes the end-user choice. As well, there should be no royalty, no discrimination, and certain guidelines should be followed to ensure that these principles are adhered to. In Table 1 we present with more detail each principle established by Perens. 
International Journal of Software Engineering \& Applications (IJSEA), Vol.2, No.1, January 2011

Table 1. Principles of open standards defined by Bruce Perens [8]

\begin{tabular}{|l|l|}
\hline \multicolumn{1}{|c|}{ Principle } & \multicolumn{1}{c|}{ Explanation } \\
\hline Availability & Open standards are available for all to read and implement. \\
\hline Maximize end-user choice & $\begin{array}{l}\text { Open standards create a fair, competitive market for } \\
\text { implementations of the standard. They do not lock the } \\
\text { customer into a particular vendor or group. }\end{array}$ \\
\hline No royalty & $\begin{array}{l}\text { Open standards are free for all to implement, with no royalty } \\
\text { or fee. Certification of compliance by the standards } \\
\text { organization may involve a fee. }\end{array}$ \\
\hline No discrimination & $\begin{array}{l}\text { Open standards and the organizations that administer them do } \\
\text { not favor one implementer over another for any reason other } \\
\text { than the technical standards compliance of a vendor's } \\
\text { implementation. Certification organizations must provide a } \\
\text { path for low or zero cost implementations to be validated, but } \\
\text { may also provide enhanced certification services. }\end{array}$ \\
\hline Extension or subset & $\begin{array}{l}\text { Implementations of open standards may be extended, or } \\
\text { offered in subset form. However, certification organizations } \\
\text { may decline to certify subset implementations, and may place } \\
\text { requirements upon extensions. }\end{array}$ \\
\hline Predatory practices & $\begin{array}{l}\text { Open standards may employ license terms that protect against } \\
\text { subversion of the standard by embrace and extend tactics. The } \\
\text { licenses attached to the standard may require the publication of } \\
\text { reference information for extensions, and a license for all } \\
\text { others to create, distribute and sell software that is compatible } \\
\text { with the extensions. An open standard may not otherwise } \\
\text { prohibit extensions. }\end{array}$ \\
\hline
\end{tabular}

The Open Standards Policy of the State of Massachusetts in USA defines open standard as specifications for systems that are publicly available and are developed by an open community and affirmed by a standards body. The European Commission's Europe Interoperability Framework (EIF) includes the requirements that open standards should be accessible either for free or at a nominal charge for usage, copying and distribution and that any patents present are to be made irrevocably available on a royalty-free basis, and there should be no constraint on the re-use of the standard.

Other organizations such as the American National Standards Institute (ANSI), the International Telegraph Union Telecommunications Standardization Sector (ITU-T) and the Business Software Alliance (BSA) have also introduced their definitions and policies on open standards. While all of these recognize that open standards have to be publicity available for implementation and participation in development by interested parties, they also recognize the inclusion of essential intellectual property rights (IPR) so long as these IPR can be made available under non-discriminatory terms and for a reasonable fee or without any fee.

Consequently, we discover that while there may be various detailed definitions and meanings given to open standards, in general, they all satisfy the following characteristics:

- Easy accessibility for all readers and users;

- Developed by a process that is open and fairly easy for anyone to participate;

- No control or linked to any specific group or vendor. 
International Journal of Software Engineering \& Applications (IJSEA), Vol.2, No.1, January 2011

Some examples of open standards in the Information Technology (IT) industry are the Transmission Control Protocol/Internet Protocol (TCP/IP) suite of networking protocols from the Internet Engineering Task Force (IETF) and the Hypertext Transfer Protocol (HTTP) service protocol from the World Wide Web Consortium (W3C) and the International Organization for Standardization (ISO).

\subsection{Benefits of open standards}

Several benefits are achieved if an organization ensures that its technological and IT implementations follow open standards whenever it is possible. Firstly, there is less possibility of being locked in by a specific technology and/or vendor. Since the specifications are known and open, it is always possible to get another party to put into operation the same solution adhering to the standards being followed. Another key benefit is that it will be easier for systems from different parties or using different technologies to interoperate with one another. Therefore, there will be enhanced data interchange and exchange. It will not be required to use the same hardware or software from a specific vendor to read or write data files. For example, if a multinational organization requires that all its offices worldwide use office software applications that can read and write files using the Open Document format defined by the Organization for the Advancement of Structured Information Standards (OASIS) [9], an individual office will have the flexibility of using whatever office software that is best suited for it and, at the same time, be able to read, write and exchange documents with other offices in the organization.

Using open standards will also offer better protection for the data files created by an application against obsolescence of the application. If the data file format used is proprietary, the user may have a tough time converting the data files to another format needed by a new application. On the other hand, if the data format follows an open standard and, hence, is known, either the new application will be able to use it as it is or it will be easier to convert the data so that the new application can use it.

If a user demands that open standards are adopted, there will be more choices available as more vendors can participate to offer solutions and it may be possible to even mix and match solutions from multiple vendors to provide best-of-breed solutions as far as possible. If open standards are followed, applications are easier to transfer from one platform to another since the technical implementation follows known guidelines and rules, and the interfaces, both internally and externally, are known. Furthermore, the skills learned from one platform or application can be used with possibly less re-training needed. This cannot be achieved with the usage in applications of proprietary standards that are not openly published and where there is inadequate information publicly available about them.

Open standards have also other abundant benefits, including enabling innovation, spreading new technology, expanding market access, boosting transparency, creating market stability, and ensuring efficiency and economic growth [10] [11]. In fact, the standards process balances change and continuity in the marketplace. A great example of this situation is the success of the Internet, which is itself built on standards. According to Vint Cerf, the Internet is fundamentally based on the existence of open and non-proprietary standards [12].

Open standards have enabled new applications that mix multiple sources of data and have created new opportunities for innovation amongst as diverse actors like governments, enterprises, SMEs and citizens. The standardization of technologies, tools or processes guarantees interoperability. Open standards are developed in a transparent and collaborative process, are available free or at a nominal cost and can be implemented royalty free, in particular regarding software interoperability standards. Additionally, open standards have 
International Journal of Software Engineering \& Applications (IJSEA), Vol.2, No.1, January 2011

demonstrable impact on software ecosystem. A recent empirical study of best practice in eGovernment mentions the use of open standards among its top seven recommendations for success [13].

However, Shapiro defends that open standards could potentially have negative effects as well, and has in a few cases constrained variety and innovation [14]. Yet, in practice this discussion seems to be rather hypothetical, and the benefits of open standards regarding choice, flexibility and innovation by far exceed such potential negative effects. Commissioner Kroes of the EU competition authority recently pointed out that opting for open standards is a very intelligent business decision indeed. He argues that for all future IT developments and procurement procedures, the Commission should promote the use of products that support open, welldocumented standards. He advocates that interoperability is a critical issue for the Commission, and usage of well established open standards is a key factor to achieve and endorse it [15].

\section{OPEN STANDARDS IN THE EUROPEAN CONTEXT}

The European Commission has for some time emphasized the important role of open standards to enable software interoperability. The initial version of i2010 strategy states that digital convergence requires devices, platforms and services to interoperate. To face this challenge, the Commission intends to use all its instruments to foster technologies that communicate, through research, promotion of open standards and support for stakeholder dialogue. The i2010 midterm review made in 2008 confirmed the importance of open standards and defends that EU should improve the framework conditions for innovation, in particular in the information society, by accelerating the setting of interoperable standards.

However, there are few specific policy activities in place to follow it up, except in cross-border situations. In 2004, the Pan-European eGovernment Programme (IDABC) in DG DIGIT issued their European Interoperability Framework (EIF 1.0) with a strict minimum definition of open standards and mandated their use in pan-European eGovernment services [16]. There, the open standards should be:

1. Adopted and maintained via an open process in which all interested parties can participate;

2. Published and available freely or at a nominal charge;

3. Made irrevocably available on a royalty free basis, even if intellectual property issues apply to patents covering all or parts of the standard;

4. Free of constraints on the re-use of the standard.

An update (EIF 2.0) was sent out for public consultation in mid-2008. EIF 2.0 draft focuses on software standards and specifications, which should take care of the critique from hardware vendors. It is sensitive to life-cycle issues, so that if one decides no potential benefit results from using open source solutions, one might limit the scope to criteria (1) and (2). That is sensible strategy, given the goal of increasing the momentum of open standards. On the other hand, criterion (3) is crucial to software interoperability and striving towards compliance is important.

The IDABC programme has also launched a Common Assessment Method for Standards and Specification (CAMSS), which aims to assist Member States in their development of eGovernment services, particularly interoperability frameworks and architectures. CAMSS builds on four principles: suitability, potential, openness and market conditions. For each 
International Journal of Software Engineering \& Applications (IJSEA), Vol.2, No.1, January 2011

criterion, a set of key questions should be asked and governments must themselves determine which are the most important elements, since all standards will be used in a particular context.

The CAMSS draft is a step in the right direction because it takes a pragmatic approach without selling out to large software vendors and giving in to hardware vendors. It introduces flexibility, urging Member States to set their own targets. The recommendations emerge from a content analysis of existing frameworks. However, the CAMSS concept is quite loose, and still a bit overwhelming to be of practical use and achieve high impact. Besides that, while the PanEuropean eGovernment Programme (IDABC) in DG DIGIT recommends open standards to Member States [16], eCommission, which is the Commission's internal IT programme, is scarcely aware of the importance of open standards [17].

The European standards policy is gradually being changed. Fortunately, the Commission already planned to introduce a set of criteria for which global standards will be eligible in the European standardization framework. Finally, it is recognized that the interoperability of national public ICT infrastructures is a requirement for a more service-oriented and competitive public sector, particularly with regard to pan-European exchange. At the national level, around twenty European governments have interoperability frameworks or action plans that favour open standards [18]. Besides that, we can find in various European countries some successful projects regarding the adoption of open standards and open source software. In the beginning of 2007, 10 major Dutch cities signed a Manifesto of the Open Cities promoted by the Dutch government [19]. Other example is the National Plan for Scientific Research, Development and Technological Innovation in Spain that included a specific budget line for Open Source Software projects, which represented 5\% of the total budget for R\&D for Information Society Technologies [20].

\section{CONNECTION BETWEen OPEn STANDARDS AND OPEn SOURCE}

\subsection{Definition of open source}

Open Source Software is software whose source code is published and made available to the public, enabling anyone to copy, modify, and redistribute the source code without paying royalties or fees [21]. This definition includes two elements:

1. Actual disclosure of the source code from the computer program;

2. The intellectual property rights license, which includes copyright license and, where applicable, patent licenses that can be used, modified and distributed without the payment of software license.

Currently, many different license regimes coexist with a wide variety of contract solutions for the actual rights and obligations of licensees. The most popular software licenses and their capabilities are presented in Table 2. 
Table 2. Rights and obligations of most popular OS software licenses [22]

\begin{tabular}{l|c|c|c|c}
$\begin{array}{c}\text { Capabilities } \\
\text { (Without Application } \\
\text { Licensing Restriction) }\end{array}$ & $\begin{array}{c}\text { GPL } \\
\text { (Linux) }\end{array}$ & $\begin{array}{c}\text { Dual-GPL } \\
\text { (MysQL) }\end{array}$ & $\begin{array}{c}\text { LGPL/MPL } \\
\text { (OpenOffice, } \\
\text { Firefox) }\end{array}$ & $\begin{array}{c}\text { Apache/BSD } \\
\text { (Apache, FreeBST) }\end{array}$ \\
\hline 1) Download & $\checkmark$ & & & \\
\hline 2) Evaluate & $\checkmark$ & & & \\
\hline 3) Deploy & $\checkmark$ & & & \\
\hline 4) Redistribute & $Q^{1}$ & $\vee^{3}$ & & \\
\hline 5) Modify & $Q^{2}$ & $Q^{2}$ & $Q^{2}$ & \\
\hline
\end{tabular}

1) Application needs to be licensed under GPL if redistributed with the GPL asset.

2) Library code modifications need to be licensed under the same license as the

originating asset.

3) Usually requires a commercial license from the copyright holder.

4) Although much more permissive than an OSI license, some BSD based licenses,

such as Apache V2, still have some copyleft materials.

The traditional options of "download" and "evaluate" are common either in open source software and proprietary software. On the other side, the deploy feature is where most misunderstandings come from and where open source differ the most from proprietary software licensing. For server technologies, all open source licenses allow users to freely build and deploy applications on top of the open source (server) asset without any restrictions on their application licensing. Restriction applies only when redistribution of the asset occurs, and typical end-user utilization does not qualify as asset redistribution.

Redistribution of an application is where GPL differs the most from other open source license types. GPL is very viral by design, and can be a great tool for an entity wishing to control the redistribution of its open source asset by assuming that many potential customers or distributors will not want to play by the GPL rules [23]. Dual-licensing (GPL + Commercial License) is often used for this purpose by allowing users to opt-out of the GPL licensing restriction if they agree to the commercial terms.

Modification of the open source asset is where BSD-like licenses (e.g., Apache, BSD, MIT) differ the most from other ones. All other open source licensing forces modifications to their asset to be submitted back with the same original license, whereas BDS/Apache-like licenses do not have such requirements [24].

\subsection{Can we combine open source and open standards?}

Open source and open standards are two distinct concepts. While they may be interrelated in some aspects, there is often confusion about their meanings and relative significance. These two concepts are at times thought to be synonymous which they are not [25]. Open source is primarily an implementation and not a specification like in the case of open standards. Open standards can be well implemented by software irrespective of whether it is open source software or other software. Furthermore, not all open source software necessarily supports 
International Journal of Software Engineering \& Applications (IJSEA), Vol.2, No.1, January 2011

widely adopted open standards or is a guarantee of interoperability between different implementations. Theorically, the modification rights and opportunities associated with open source are not guarantee of continued interoperability as they permit the creation of new versions which then quite possibly can be incompatible.

The advantage of OSS is that the disclosure of source code allows any user to modify the code to ensure compliance with open standards for interoperability provided, of course, that the user complies with the license conditions relevant to the code the user has modified. Market experience with OSS to date does not demonstrate significant, irresolvable interoperability problems with the most widely used popular OSS applications. One rational explanation for this is that open source developers are gathering together to solve generic problems they share. Open source is not only a piece of software but it is also a process to build and license code in order to solve common shared problems such as infrastructure problems.

A natural source for open source developers is open standards. These developers natively implement in the OSS and the result is a de facto support of open standards in OSS. The process is not divergent to support of open standards in non-OSS products, however, the proponents of OSS model believe the OSS communities have a steady preference to open standard implementation. It is entirely possible for a feature in a non-OSS software (often called proprietary software) to be implemented following an open standard. Open standards are neutral with regard to software licensing or business models and so it is equally possible for an open standard to be implemented in proprietary software as it is in OSS. For example, proprietary software like the Microsoft Windows operating system can still implement the TCP/IP networking protocols following the open standards from IETF and be compliant with them.

The open source licensing model enables distribution and usage of software without any or minor restrictions. This network effect is capable of accelerating propagation of standard usage and thereby can be a contributing factor to better interoperability. The accessibility of the source code and the design information as well as the rights to modify, onward develop and distribute OSS support reusability of good implementations. Also, the community of participants working with OSS may promote open debate resulting in an increased recognition of the benefits of various solutions and such debate may accelerate the adoption of solutions that are popular among the OSS participants. These characteristics of OSS support evolution of robust solutions are often a significant boost to the market adoption of open standards, in addition to the customer-driven incentives for interoperability and open standards.

Open source software enhances trust in interoperability through transparency. When source code and compiler are accessible, users are able to verify that the software interoperates as it should and organizations have a solution whose security, privacy and transparency is not dependent on actions of and are continued support by their suppliers. In addition, the open source rights model supports platform portability, which allows the software to easily adapt to different operating systems or other platform elements. This can support wide dissemination on many platforms resulting in wide deployment of interoperable implementations.

OSS can play a useful role in popularizing an open standard. An OSS implementation of a standard usually results in an open and free-working reference implementation. The availability of an OSS implementation will spur quicker adoption and acceptance of the standard as everyone has easy access to the implementation of the standard and so can try and test it out. A very good example of this is the Internet HTTP standard. One reason why this service became universally accepted is that very early on there were free and open implementations of both the HTTP server (e.g., National Center of Supercomputing Applications or NCSA HTTPd, Apache) and client (e.g., NCSA Mosaic). 
International Journal of Software Engineering \& Applications (IJSEA), Vol.2, No.1, January 2011

Open source software can also have important economic and social impacts. OSS brings the opportunity to citizens to create and add value. Traditional access to technology alone limits them to the role of passive consumers in the knowledge community, while the ability to create transforms them into active participants. OSS provides an excellent training environment that enables this ability to create and increases the earning capacity of community participants without any explicit investment in training and perhaps a novel form of technology transfer.

This in turn makes it more attractive to governments and policy makers. Countries around the world, despite of their wealth and economical power, are trying to bring citizens into the Information Society and provide electronic access to government services. Many of them, in particular the European Union, are considering and implementing open source solutions as a cost-effective means of doing so. Many more see an inherent injustice in requiring citizens and businesses to buy software from specific vendors in order to communicate with the government, and are looking at open standards, which allow products from different producers of open source or proprietary software to work together.

\section{Conclusions}

Government and companies are increasing their efforts to meet the growing challenge of interoperability between new technologies, applications or hardware devices. In truth, interoperability has a major positive impact on innovation, growth and competitiveness. While interoperability may not be a primary concern when new technologies are launched in market and only used by a small group of users, accomplishment of broad-based interoperability based on open standard specifications become increasingly more important as a larger market develops.

Open standards are even more important in the actual information age of IT and communications convergence over the Internet. No single technology, group or vendor can provide all features and, as a result, interoperability in a heterogeneous environment is required more than ever. It is only by strict adherence to standards and specifications that a high degree of interoperability can be achieved. Standards that are open and non-discriminatory are preferred because there is no dependence on any single entity, all types of products can implement them and all interested parties can partake in their development. Besides that, open standards have also proven to be an important facilitator for innovation. By providing an agreed, reliable and globally valid base of technology, open standards allow innovators to develop highly competitive, innovative technologies and solutions "on top" of the standard.

The introduction to the market of open standards is facilitated when it is used with open source software. Open source software can support reusability of good implementations and is frequently an important enhance to the market adoption of open standards. Open standards can be well implemented by software irrespective of whether it is open source software or proprietary software. However, access to source code can also improve trust in interoperability through transparency and it also may support platform portability. Moreover, open source software provides relevant significant cost advantages and the process of learning and adapting software enables users to become active players in the knowledge society.

\section{REFERENCES}

[1] Raza. A. \& Capretz, L. (2010) “Contributors' Preference in Open Source Software Usability: An Empirical Study”, International Journal of Software Engineering \& Applications, Vol. 1, No. 2, pp. 45-64. 
International Journal of Software Engineering \& Applications (IJSEA), Vol.2, No.1, January 2011

[3] Deshpande, A. \& Riechle, D. (2008) "The Total Growth of Open Source", Proceedings of the Fourth Conference on Open Source Systems, Spring Verlang, pp. 197-209.

[4] Walli, S., Gynn, D. \& Rotz, V. (2007) “The Growth of Open Source Software in Organizations”, Optaros White Paper, pp. 5-11.

[5] IDC (2007) Worldwide Open Source Software Business Models 2007-2011 Forecast: A Preliminary View, IDC Publishers.

[6] SIIA (2006) "Packaged Software Industry Revenue and Growth", Software and Information Industry Association White Papers, pp. 24-27.

[7] Hoe, N. \& Quinn, P. (2006) Free/Open Source Software and Open Standards, UNDP-APDIP, Elsevier.

[8] Perens, B. (2009) “Open Standards Principles and Practice”, Available at: http://perens.com/OpenStandards/Definition.html

[9] OASIS (2005) “Open Document Format for Office Applications (OpenDocument) v1.0”, Available at: http://www.oasis-open.org/specs/index.php\#opendocumentv1.0

[10] Blind, K. (2004) The Economics of Standards, Edward Elgar Publishers.

[11] Weitzel, T. (2004) Economics of Standards in Information Networks, Physica Verlag Publishers.

[12] Cerf, V. (2006) "Hearing on Network Neutrality", Available at: http://commerce.senate.gov/pdf/cerf-020706.pdf

[13] Undheim, T. \& Friedrich, J. (2008) "The Momentum of Open Standards - A Pragmatic Approach to Software Interoperability", European Journal of ePractice, Vol. 5, No. 1, pp. 1-13.

[14] Shapiro, C. (2001) Setting Compatibility Standards: Cooperation or Collusion, Oxford University Press.

[15] Kroes, N. (2008) "Being Open about Open Standards", Europa Press Releases, Vol. August, No. 317, pp. 1-6.

[16] IDABC (2008) "Documentation on the Promotion of Open Document Exchange Format", Available at: http://ec.europa.eu/idabc/en/document/3439/5585

[17] Cassell, M. (2008) "Why Governments Innovate: Adoption and Implementation of Open Source Software by Four European Cities”, International Public Management Journal, Vol. 11, No. 2, pp. 193-213.

[18] EU (2007) "European eGovernment 2005-2007 - Taking Stock of Good Practice and Progress towards Implementation on the i2010 eGovernment Action Plan", Available at: http://www.epractice.eu/document/3927

[19] Vervloesem, K. (2007) “Ten Big Dutch Cities Demand Open Standards”, Available at: http://www.linux.com/archive/feature/59497

[20] CSIS (2008) "Government Open Source Policies", Center for Strategic and International Studies White Papers, pp. 6-28.

[21] OSI (2010) "OSI: The Open Source Definition”, Available at: http://www.opensource.org/docs/definition.php

[22] Chone, J. (2008) "Which Open Source License?”, Available at: http://www.bitsandbuzz.com/article/which-open-source-license/

[23] Kaminski, H. \& Perry, M. (2007) “Open Source Software Licensing Patterns”, Available at: http://ir.lib.uwo.ca/cgi/viewcontent.cgi?article $=1009 \&$ context $=$ csdpub

[24] Manabe, Y., Hayase, Y. \& Inoue, K. (2010) "Evolutional Analysis of Licenses in FOSS", Proceedings of the Joint ERCIM Workshop on Software Evolution and International Workshop on Principles of Software Evolution, pp. 83-87. 
International Journal of Software Engineering \& Applications (IJSEA), Vol.2, No.1, January 2011

[25] Cerri, D. \& Fuggetta, A. (2007) "Open Standards, Open Formats, and Open Source", Journal of Systems and Software, Vol. 80, No. 11, pp. 1930-1937.

Authors

Fernando Almeida has a $\mathrm{PhD}$. degree in Electronic and Computer Engineering and a master degree in the field of innovation and entrepreneurship. His major research interests are in the field of Open Source software, business models and open innovation policies.

José Oliveira has a $\mathrm{PhD}$. degree in Electronic and Computer Engineering. His major research interests are in the field of telecommunications economy, business models for Web 2.0 platforms and software architectures.

José Cruz has a $\mathrm{PhD}$. degree in Informatics Engineering. His major research interests are in the field of Open Source software, AGILE software development techniques, IT security and parallel computing.

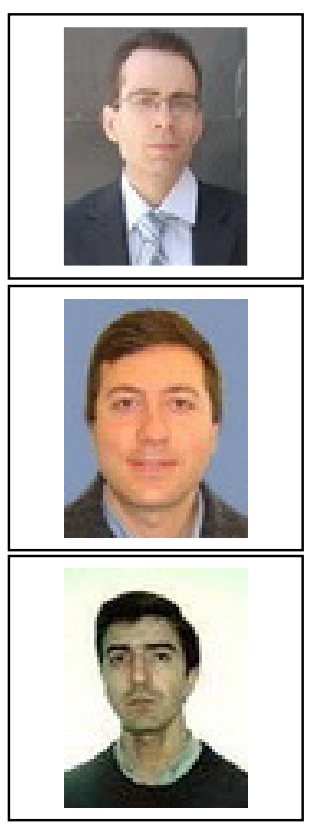

